\title{
OCCURRENCE OF EXTREME SOLAR PARTICLE EVENTS: ASSESSMENT FROM HISTORICAL PROXY DATA
}

\author{
ILYA G. Usoskin ${ }^{1}$ and Gennady A. Kovaltsov ${ }^{2}$ \\ ${ }^{1}$ Sodankylä Geophysical Observatory (Oulu unit) and Department of Physical Sciences, University of Oulu, \\ FIN-90014 Oulu, Finland; ilya.usoskin@oulu.fi \\ ${ }^{2}$ Ioffe Physical-Technical Institute of RAS, 194021 St. Petersburg, Russia \\ Received 2012 March 30; accepted 2012 July 24; published 2012 September 6
}

\begin{abstract}
The probability of occurrence of extreme solar particle events (SPEs) with proton fluence $(>30 \mathrm{MeV}) F_{30} \geqslant$ $10^{10} \mathrm{~cm}^{-2}$ is evaluated based on data on the cosmogenic isotopes ${ }^{14} \mathrm{C}$ and ${ }^{10} \mathrm{Be}$ in terrestrial archives covering centennial-millennial timescales. Four potential candidates with $F_{30}=(1-1.5) \times 10^{10} \mathrm{~cm}^{-2}$ and no events with $F_{30}>2 \times 10^{10} \mathrm{~cm}^{-2}$ are identified since $1400 \mathrm{AD}$ in the annually resolved ${ }^{10} \mathrm{Be}$ data. A strong SPE related to the Carrington flare of $1859 \mathrm{AD}$ is not supported by the data. For the last 11,400 years, $19 \mathrm{SPE}$ candidates with $F_{30}=(1-3) \times 10^{10} \mathrm{~cm}^{-2}$ are found and clearly no event with $F_{30}>5 \times 10^{10} \mathrm{~cm}^{-2}$ (50 times the SPE of 1956 February 23) has occurred. These values serve as observational upper limits on the strength of SPEs on the timescale of tens of millennia. Two events, ca. 780 and $1460 \mathrm{AD}$, appear in different data series making them strong candidates for extreme SPEs. We build a distribution of the occurrence probability of extreme SPEs, providing a new strict observational constraint. Practical limits can be set as $F_{30} \approx 1,2-3$, and $5 \times 10^{10} \mathrm{~cm}^{-2}$ for occurrence probabilities $\approx 10^{-2}, 10^{-3}$, and $10^{-4} \mathrm{yr}^{-1}$, respectively. Because of the uncertainties, our results should be interpreted as a conservative upper limit on the SPE occurrence near Earth. The mean solar energetic particle (SEP) flux is evaluated as $\approx 40\left(\mathrm{~cm}^{2} \mathrm{~s}\right)^{-1}$, in agreement with estimates from lunar rocks. On average, extreme SPEs contribute about $10 \%$ to the total SEP fluence.
\end{abstract}

Key words: solar-terrestrial relations - Sun: heliosphere - Sun: particle emission

\section{INTRODUCTION}

Sporadic energy releases on the Sun can accelerate solar energetic particles (SEPs) in the corona and interplanetary medium. Such phenomena often lead to solar particle events (SPEs) observed at Earth; this is an important factor in solar-terrestrial relations, and specifically space weather (Mewaldt 2006; Vainio et al. 2009). A typical quantity for an SPE is the fluence of SEPs with energy above $30 \mathrm{MeV}, F_{30}$. We have sufficient knowledge of SPEs over the space era since the mid-1950s (Smart et al. 2006), with only several events with $F_{30}=(1-10) \times 10^{9} \mathrm{~cm}^{-2}$ and hundreds of weaker SPEs observed. However, it is important to know, both for purely theoretical aspects of solar/stellar physics and for technical applications, the statistics of extreme SPEs with $F_{30}>10^{10} \mathrm{~cm}^{-2}$. Such a study is possible only by using indirect proxy data. Some estimates have been obtained from measurements of cosmogenic isotopes in lunar rocks (Nishiizumi et al. 2009), but this gives only the average flux of SEPs over a very long scale without extracting individual SPEs. A list of potential SPEs over the last 500 years was proposed based on nitrate records in polar ice (McCracken et al. 2001), but this result is heavily debated (Wolff et al. 2008, 2012). Data on the cosmogenic nuclides ${ }^{14} \mathrm{C}$ and ${ }^{10} \mathrm{Be}$ measured in terrestrial archives may provide information on SPEs in the past (Lingenfelter \& Hudson 1980; Usoskin et al. 2006; Webber et al. 2007), but this possibility has not been fully explored previously. Accordingly, the probability of extreme SPEs has remained exceedingly uncertain (Hudson 2010).

Here we establish a solid observational constraint on the distribution of extreme SPEs using presently available data sets on cosmogenic isotopes $\left({ }^{14} \mathrm{C}\right.$ and $\left.{ }^{10} \mathrm{Be}\right)$ measured in terrestrial archives with sufficient time resolution and quality, and modern models of their production in the atmosphere. We note that the result presented is based on terrestrial data and may not well represent the occurrence of solar events, whose geo-efficiency is also affected by the relative Sun-Earth attitude.

\section{DATA SETS AND THE METHOD}

\subsection{Data}

The data sets used are as follows:

1. IntCal09 $\Delta{ }^{14} \mathrm{C}$ global series: $11000 \mathrm{BC}-1900 \mathrm{AD}, 5$ year time resolution (Reimer et al. 2009)

2. SB93 $\Delta^{14}$ C global annual series: $1511-1900$ AD (Stuiver \& Braziunas 1993)

3. Dye $3{ }^{10} \mathrm{Be}$ Greenland annual series: 1424-1985 AD (McCracken et al. 2004)

4. NGRIP ${ }^{10} \mathrm{Be}$ Greenland annual series: $1389-1994$ AD (Berggren et al. 2009)

5. SP ${ }^{10}$ Be South Pole Antarctic series: $850-1950$ AD, quasidecadal (Raisbeck et al. 1990; Bard et al. 1997)

6. $\mathrm{DF}{ }^{10} \mathrm{Be}$ Dome Fuji Antarctic series: $695-1880 \mathrm{AD}$, quasidecadal (Horiuchi et al. 2008)

7. GRIP ${ }^{10} \mathrm{Be}$ Greenland series: $7380 \mathrm{BC}-1640 \mathrm{AD}$, quasidecadal (Yiou et al. 1997; Vonmoos et al. 2006)

8. M12 $\Delta^{14} \mathrm{C}$ Japanese annual series: $750-820$ AD, annual/biannual (Miyake et al. 2012)

\subsection{Model Computations}

In order to evaluate possible SPE signatures in the data, we used model computations of the isotope production by energetic particles, assuming instant injection of SEPs into the atmosphere and calculating the expected isotope response in terrestrial archives.

As the reference event, we considered an extreme SPE with a very hard spectrum (Tylka \& Dietrich 2009; Usoskin et al. 2011) from 1956 February 23 (SPE56; Meyer et al. 1956). While SPE56 was the strongest observed ground-level enhancement (GLE), $>4000 \%$ in the count rate of the Leeds NM, it had relatively modest fluence, $F_{30}=10^{9} \mathrm{~cm}^{-2}$ (Shea \& Smart 1990). However, sometimes SPEs with large fluence but a soft spectrum occur, e.g., a modest (only 10\% at the polar Oulu 


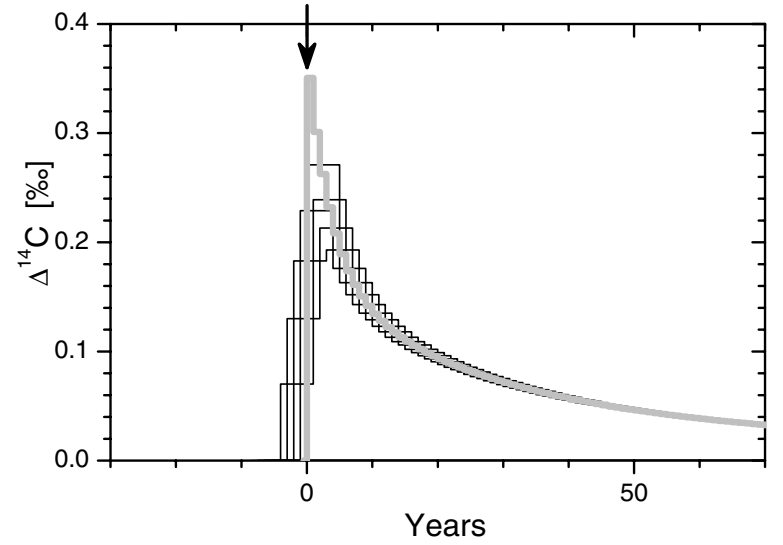

Figure 1. Response of the relative tropospheric concentration $\Delta{ }^{14} \mathrm{C}$ to SEP56 $(t=0$ shown by the arrow), with annual (gray curve) and 5 year (black) time resolutions, depending on the 5 year bin boundaries.

NM) GLE event of 1972 August 4 (SPE72) with high fluence, $F_{30}=5 \times 10^{9} \mathrm{~cm}^{-2}$. Since cosmogenic isotopes are produced by the most energetic part of the SEP energy spectra $(>1 \mathrm{GeV})$, we consider here the SPE56 scenario.

The response of ${ }^{10} \mathrm{Be}$ to SPEs was calculated similar to Usoskin et al. (2006) but using an updated ${ }^{10} \mathrm{Be}$ yield function (Kovaltsov \& Usoskin 2010), the corresponding geomagnetic model, and an intermediate atmospheric mixing model (polar tropospheric and hemispheric stratospheric mixing — see Field et al. 2006; Heikkilä et al. 2009). The calculated ${ }^{10} \mathrm{Be}$ production is $7.5 \times 10^{4}$ atoms $\mathrm{cm}^{-2}$ for the SPE56 scenario. Because of the ${ }^{10} \mathrm{Be}$ stratospheric residence time (Heikkilä et al. 2009), a possible SPE peak in ${ }^{10} \mathrm{Be}$ data can be $2-3$ years long. Since present models cannot convert the ${ }^{10} \mathrm{Be}$ production into concentrations in ice, they are typically assumed to be directly proportional to each other (e.g., McCracken et al. 2004). For the normalization we used the reference period 1850-1900 AD with moderate solar activity: the galactic cosmic ray (GCR) modulation potential $443 \mathrm{MV}$ (Alanko-Huotari et al. 2007) was close to the mean Holocene value (Usoskin et al. 2007). Accordingly, the reference period ${ }^{10} \mathrm{Be}$ production was $\langle Q\rangle=$ $3.45 \times 10^{-2}$ atoms cm $\mathrm{cm}^{-1}$ or $1.1 \times 10^{6}$ atoms cm$^{-2} \mathrm{yr}^{-1}$. This value was scaled to the mean measured concentration for the reference period in each ice core $(1.025,1.86,3.47$, and $2.76 \times 10^{4}$ atoms $\mathrm{g}^{-1}$ for the Dye3, NGRIP, SP, and DF series, respectively). The GRIP series was normalized to the entire interval.

The expected radiocarbon response of $\Delta^{14} \mathrm{C}$ was calculated in two steps. First, the global production $Q$ was computed using a new production model (Kovaltsov et al. 2012), yielding $2.9 \times$ $10^{6}$ atoms $\mathrm{cm}^{-2}$ for SPE56. Next, the instant atmospheric injection of ${ }^{14} \mathrm{C}(Q)$ was passed through a 5-box carbon cycle model (Damon \& Peristykh 2004), and the tropospheric $\Delta^{14} \mathrm{C}$ was calculated. Figure 1 shows the expected response in $\Delta^{14} \mathrm{C}$ for SPE56, which is extended over decades and is characterized by a sharp increase and exponential decay; the peak's FWHM is $15-20$ years. Accordingly, we look for a signature like that in the $\Delta^{14} \mathrm{C}$ data. The SPE56 peak in the annual (5 year) $\Delta^{14} \mathrm{C}$ data is $0.35(0.19-0.27)$ permille, viz., below measurement errors of $\approx 2$ permille. Therefore, an SPE needs to be a factor $X_{\text {SPE56 }}=10$ greater than SPE56 to be observable in the radiocarbon data.

An SPE72-type soft event would require a 40 times larger $F_{30}$, with respect to SPE56, to produce the same amount of cosmogenic isotopes. Accordingly, the estimates obtained with
Table 1

Identified SPE Candidates: Approximate Year, Data Set, and Fluence $F_{30}\left(\mathrm{~cm}^{-2}\right)$ Evaluated for the SPE56 Scenario

\begin{tabular}{|c|c|c|}
\hline SPE Year & Series & $F_{30}\left(X_{\text {SPE56 }}\right)$ \\
\hline \multirow[t]{2}{*}{$\overline{1460-1462 \mathrm{AD}^{\mathrm{a}}}$} & NGRIP(1460) & $1.5 \times 10^{10}(15)$ \\
\hline & Dye3 (1462) & $9.7 \times 10^{9}(10)$ \\
\hline $1505 \mathrm{AD}$ & Dye 3 & $1.3 \times 10^{10}(13)$ \\
\hline $1719 \mathrm{AD}$ & NGRIP & $1 \times 10^{10}(10)$ \\
\hline $1810 \mathrm{AD}$ & NGRIP & $1 \times 10^{10}(10)$ \\
\hline 8910 BC & IntCal09 & $2.0 \times 10^{10}(20)$ \\
\hline $8155 \mathrm{BC}$ & IntCal09 & $1.3 \times 10^{10}(13)$ \\
\hline $8085 \mathrm{BC}$ & IntCal09 & $1.5 \times 10^{10}(15)$ \\
\hline $7930 \mathrm{BC}$ & IntCal09 & $1.3 \times 10^{10}(13)$ \\
\hline $7570 \mathrm{BC}$ & IntCal09 & $2.0 \times 10^{10}(20)$ \\
\hline $7455 \mathrm{BC}$ & IntCal09 & $1.5 \times 10^{10}(15)$ \\
\hline 6940 BC & IntCal09 & $1.1 \times 10^{10}(11)$ \\
\hline $6585 \mathrm{BC}$ & IntCal09 & $1.7 \times 10^{10}(17)$ \\
\hline $5835 \mathrm{BC}$ & IntCal09 & $1.5 \times 10^{10}(15)$ \\
\hline $5165 \mathrm{BC}$ & GRIP & $2.4 \times 10^{10}(24)$ \\
\hline $4680 \mathrm{BC}$ & IntCal09 & $1.6 \times 10^{10}(16)$ \\
\hline $3260 \mathrm{BC}$ & IntCal09 & $2.4 \times 10^{10}(24)$ \\
\hline $2615 \mathrm{BC}$ & IntCal09 & $1.2 \times 10^{10}(12)$ \\
\hline $2225 \mathrm{BC}$ & IntCa109 & $1.2 \times 10^{10}(12)$ \\
\hline $1485 \mathrm{BC}$ & IntCal09 & $2.0 \times 10^{10}(20)$ \\
\hline $95 \mathrm{AD}$ & GRIP & $2.6 \times 10^{10}(26)$ \\
\hline $265 \mathrm{AD}$ & IntCa109 & $2.0 \times 10^{10}(20)$ \\
\hline \multirow[t]{3}{*}{$780 \mathrm{AD}^{\mathrm{a}}$} & IntCal09 & $2.4 \times 10^{10}(24)$ \\
\hline & M12 & $4 \times 10^{10}(40)^{b}$ \\
\hline & $\mathrm{DF}$ & $4.5 \times 10^{10}(45)^{b}$ \\
\hline $1455 \mathrm{AD}^{\mathrm{a}}$ & SP & $7 \times 10^{10}(70)^{b}$ \\
\hline
\end{tabular}

Notes. Scaling to SPE56 is given in parentheses.

${ }^{a}$ Discussed separately.

b Overestimate.

the reference SPE56 spectrum should be enhanced 40-fold to correspond to a soft-spectrum SPE72 scenario.

\section{EVALUATING SEP FLUENCE IN THE PAST}

First, we analyzed data series with annual resolution (2-4 in Section 2.1), looking for potential signatures of SPEs.

For the NGRIP series we looked for peaks with magnitude $\geqslant 1.3 \times 10^{4}$ atoms $\mathrm{g}^{-1}$ and duration $\leqslant 3$ years. Seven candidates were selected: $1436,1460,1650,1719,1810,1816$, and $1965 \mathrm{AD}$. For the ${ }^{10} \mathrm{Be}$ Dye3 series, five candidates (magnitude $\geqslant 0.6 \times 10^{4}$ atoms $\mathrm{g}^{-1}$, duration $\leqslant 3$ years) were selected: $1462,1479,1505,1512$, and $1603 \mathrm{AD}$. For the ${ }^{14} \mathrm{C}$ SB93 series, no suitable candidates (peak with sharp rise and gradual decay) were found. Since the candidates found do not coincide in time, we performed a cross-check. For each candidate found in series $A$ we calculated, using the model (Section 2.2), the corresponding isotope production $Q$ and the expected response in series $B$, and checked if they were consistent with the data within \pm 2 years and vice versa. For example, a 1436 AD peak in the NGRIP series must be accompanied by a strong peak in the Dye3 series, which was not observed. Also, an NGRIP 1965 AD peak is rejected by direct cosmic ray observations. Using this cross-check, seven candidates can be excluded: 1436, 1650,1816 , and 1965 AD in NGRIP and 1479, 1512, and 1603 $\mathrm{AD}$ in the Dye3 series. The SB93 series is insensitive to checking the ${ }^{10} \mathrm{Be}$-based candidates. Finally, five peaks pass through the check as potential SPE signatures (first block in Table 1).

Only one candidate (1460-1462 AD) is present in both series (discussed later). 


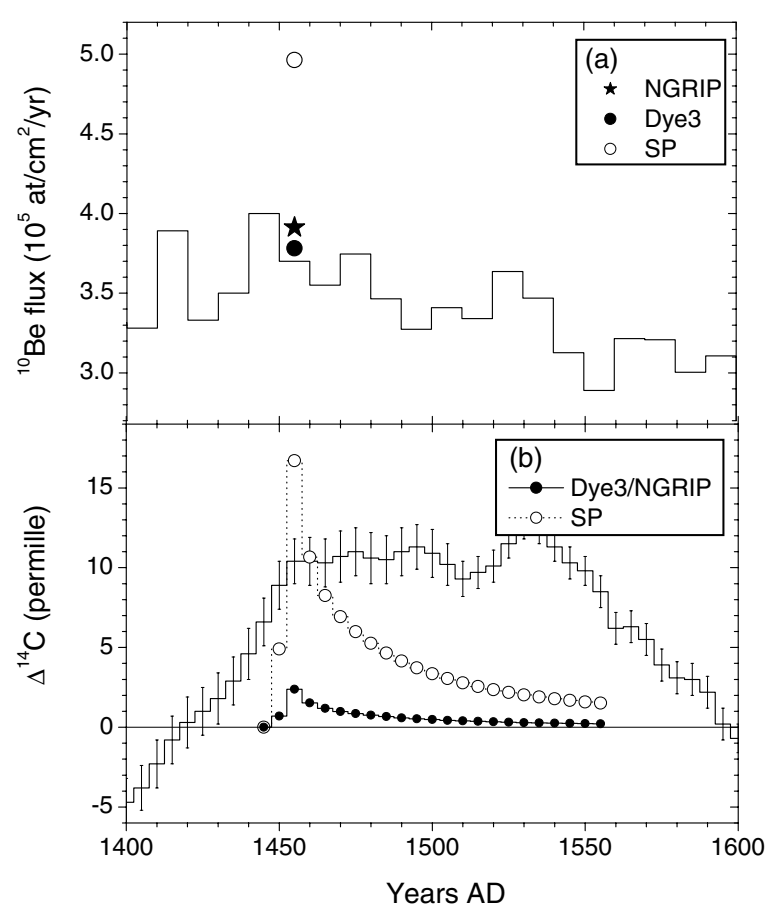

Figure 2. Test of a possible SPE ca. $1460 \mathrm{AD}$. (a) $\mathrm{DF}{ }^{10} \mathrm{Be}$ series, with points corresponding to the SEP production estimated from the NGRIP, Dye3, and SP data series (see the legend). (b) IntCal09 $\Delta{ }^{14} \mathrm{C}$ series along with the expected signal estimated from the Dye3/NGRIP and SP data series.

Next, we considered data with rougher time resolutions. In each of the ${ }^{10} \mathrm{Be}$ data series (5-7 in Section 2.1) with quasidecadal resolution, we searched for distinctive single peaks. Two candidates were identified in the GRIP series: ca. 5165 BC and $95 \mathrm{AD}$. One candidate was identified in the SP series: ca. $1455 \mathrm{AD}$, probably related to the ca. 1460 event in the NGRIP and Dye 3 annual series. Two candidates were identified in the DF series: ca. $780 \mathrm{AD}$ and $1805 \mathrm{AD}$. A search for signatures (sharp $\leqslant 10$ year increase, FWHM $15-30$ years, magnitude $>2$ permille) in the IntCal09 ${ }^{14} \mathrm{C}$ series yields many candidates (see an example in Figure 3(a)). We also made a cross-check of all the candidates between different series. For instance, the $1805 \mathrm{AD}$ peak in the DF series was rejected based on the annual NGRIP and Dye3 data. About half of the candidates in the IntCal09 series after $7000 \mathrm{BC}$ were rejected based on the ${ }^{10} \mathrm{Be}$ data. Therefore, half of the identified candidates before $7000 \mathrm{BC}$ may also be spurious.

Finally, in Table 1 we list the candidates passing the crosscheck. One can see that 10 times SPE56, $X_{\text {SPE56 }}=10$, is the detection limit in cosmogenic isotope data. Only two candidates appear in more than one series: ca. $780 \mathrm{AD}$ and $1460 \mathrm{AD}$, which are discussed in detail below.

\subsection{Event of ca. $1460 A D$}

Several series analyzed show a significant peak around 1460 $\mathrm{AD}$ (Table 1). This was noticed previously and ascribed to a very strong SPE or to a supernova explosion (e.g., Berggren 2009; McCracken et al. 2004; Delaygue \& Bard 2011). We assume here that it is an SPE signature and evaluate its parameters. Annual NGRIP and Dye3 series depict distinct peaks in 1460 and $1462 \mathrm{AD}$, respectively, which roughly agree with each other, and require $F_{30} \approx 10^{10}$ protons $\mathrm{cm}^{-2}$ for the SPE56 scenario. The more roughly resolved SP series depicts a very strong peak ca. $1455 \mathrm{AD}$, requiring a fluence seven times greater than that

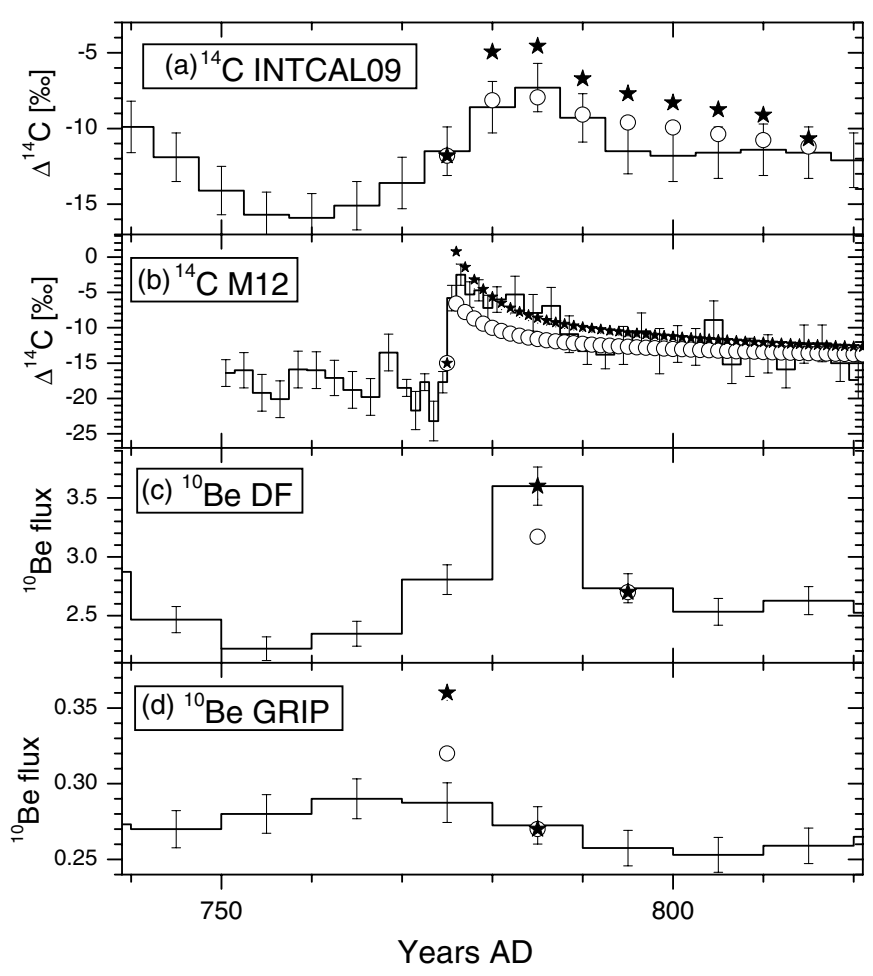

Figure 3. Test of a possible SPE ca. 780 AD. Open dots and black stars correspond to the modeled response to an SEP event equal to $X_{\text {SPE56 }}=24$ and $X_{\text {SPE56 }}=45$, respectively. (a) IntCal09 $\Delta^{14} \mathrm{C}$ data series. (b) $\mathrm{M} 12 \Delta^{14} \mathrm{C}$ data series. (c) $\mathrm{DF}{ }^{10} \mathrm{Be}$ data series. (d) GRIP ${ }^{10} \mathrm{Be}$ data series.

implied from the NGRIP/Dye3 data. None of the other series (GRIP, DF, or IntCal09) show peaks around that date allowing for evaluation of the upper limit. Figure 2(a) shows a crosscheck of this event versus the DF series. While the event strength evaluated from the NGRIP and Dye3 series is consistent with no clear signal in the DF data, the huge fluence implied from the SP data contradicts the DF data (cf. Delaygue \& Bard 2011). A similar analysis of the GRIP data also suggests that the SP peak is too high. Figure 2(b) shows the IntCal09 data along with signals expected from the NGRIP/Dye3 and SP data, respectively, for the ca. $1460 \mathrm{AD}$ candidate. Because of the steep gradient in $\Delta^{14} \mathrm{C}$ in $1430-1470$, it is impossible to distinguish a small 2.4 permille peak implied by the Dye3/NGRIP data, but the high signal implied by the SP peak apparently contradicts the data. Thus, while all the data sets analyzed are consistent with a hypothesis of a strong SPE ca. $1460 \mathrm{AD}$, its fluence is likely about $10^{10}$ protons $\mathrm{cm}^{-2}$ for the SPE56 scenario. The very high fluence implied by the SP data contradicts the other data sets.

\subsection{Event of ca. $780 A D$}

Another interesting candidate for a strong SPE is ca. 780 $\mathrm{AD}$ as manifested through distinct peaks in the DF, IntCal09, and M12 data series (Figure 3). According to these data sets, the event was 25-50 times stronger than SPE56 with fluence $F_{30}=(2-5) \times 10^{10}$ protons $\mathrm{cm}^{-2}$. The best fit for the IntCal09 data (Figure 3(a)) is obtained for an $X_{\text {SPE56 }}=24$ event starting ca. 780 AD. The M12 data (Figure 3(b)) are a better fit with an $X_{\mathrm{SPE} 56}=40$ event starting in $775 \mathrm{AD}$, while the $\mathrm{DF}$ data (Figure 3(c)) are consistent with an $X_{\mathrm{SPE} 56}=45$ event that occurred between 780 and 790 AD. However, the GRIP series (Figure 3(d)) depicts no peak in that period, which indicates that a fluence greater than $F_{30}=3 \times 10^{10}$ protons cm $\mathrm{cm}^{-2}$ is inconsistent at the 0.03 significance level. Applying the 


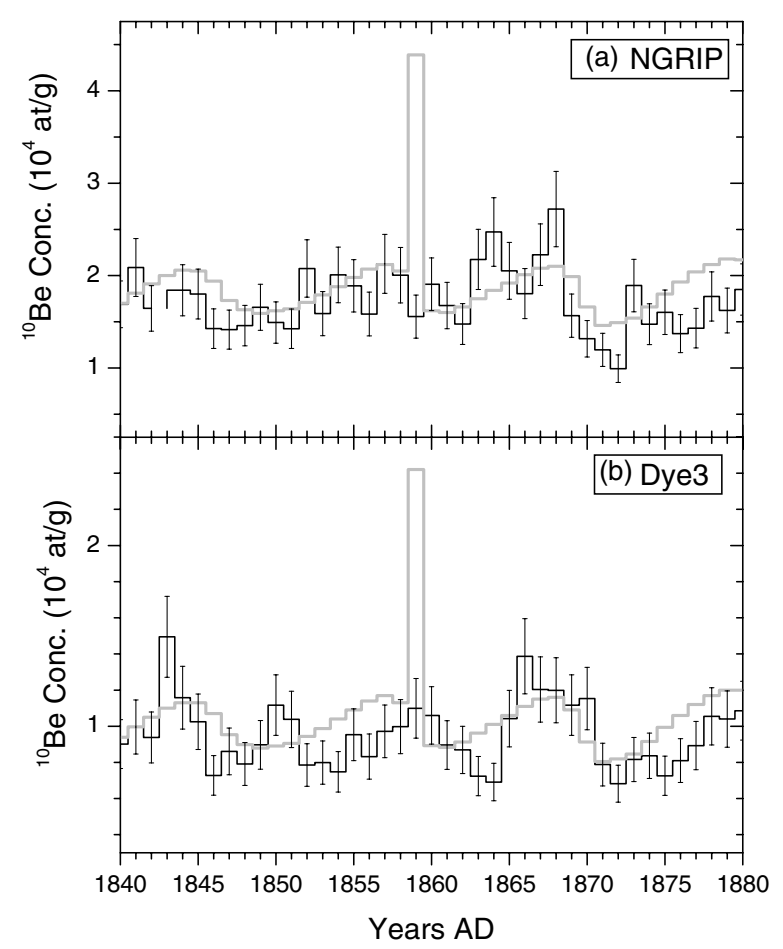

Figure 4. Testing a possible SEP event ca. 1859 AD. The gray line depicts a model GCR signal plus the addition of the annual production due to the Carrington 1859 AD event according to McCracken et al. (2001). (a) Time series of the annual NGRIP ${ }^{10} \mathrm{Be}$ data, with $15 \%$ error bars. (b) Time series of the annual Dye $3{ }^{10} \mathrm{Be}$ data, with $15 \%$ error bars.

cross-check, we found that the fluence implied by the IntCal09 data $\left(X_{\text {SPE56 }}=24-\right.$ see Table 1$)$ is consistent with the GRIP data, while both $X_{\text {SPE56 }}=40$ and 45 based on the DF and M12 data, respectively, yield too high a peak in the GRIP series. Accordingly, we conclude that the event of $780 \mathrm{AD}$ is a possible candidate for a strong SPE with a consensus value of $F_{30} \approx 3 \times 10^{10}$ protons cm $\mathrm{cm}^{-2}$.

\subsection{Carrington Event of 1859 AD}

The Carrington event of $1859 \mathrm{AD}$ is often considered to be an extreme SPE, with $F_{30}=1.8 \times 10^{10} \mathrm{~cm}^{-2}$ as estimated from the nitrate record in the Greenland Summit core (Dreschhoff \& Zeller 1998; McCracken et al. 2001; Shea et al. 2006). Although none of the series analyzed here depict a peak around 1859 , we checked if this proposed SPE is consistent with the cosmogenic data. Using the GCR modulation reconstruction (Alanko-Huotari et al. 2007) and the ${ }^{10}$ Be production model (Kovaltsov \& Usoskin 2010), we calculated the expected ${ }^{10} \mathrm{Be}$ concentration along with the additional production for $1859 \mathrm{AD}$, applying SEP parameters for the Carrington SPE according to Section 8 of McCracken et al. (2001). The model computation was then confronted with the annual data of two Greenland series, NGRIP and Dye3, in Figure 4. The ${ }^{10}$ Be peak, expected from the nitrate data, is too strong, contradicting the observed data in the two Greenland sites. Radiocarbon data and decadal ${ }^{10} \mathrm{Be}$ series cannot resolve the Carrington peak. Therefore we conclude that the cosmogenic data do not support the hypothesis of a very strong SPE related to the Carrington flare.

\section{DISCUSSION}

We propose, by analyzing data from different cosmogenic isotope records and performing cross-checks, a list of candidates

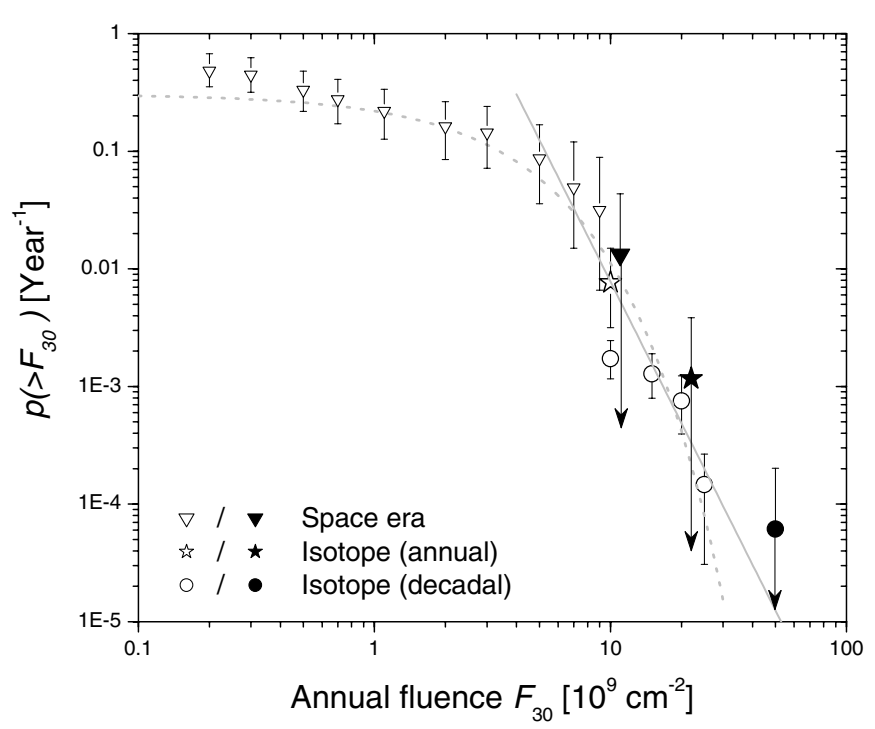

Figure 5. Probability of occurrence of the annual fluence ( $>30 \mathrm{MeV}$ ) exceeding the given value $F_{30}$, as evaluated from the data for the space era 1956-2008 (triangles), cosmogenic isotope annual data (stars), and cosmogenic isotope decadal data (circles). Open symbols correspond to the statistics of "observed" data, while filled symbols denote the conservative upper limit (no events greater than $X$ observed during $Y$ years). Error bars correspond to the $90 \%$ confidence level evaluated assuming Poisson statistics. Gray dotted and solid lines depict the best-fit exponent and power-law approximations for $F_{30}>7 \times 10^{9}$ protons $\mathrm{cm}^{-2}$.

for strong SPEs in the past (Table 1). The list is somewhat uncertain, as some events may be missing or some spurious peaks falsely identified. However, the result is quite consistent in a statistical sense.

Four candidates with an $X_{\text {SPE56 }}$ factor of 10-15 are identified in the annual ${ }^{10} \mathrm{Be}$ series NGRIP and Dye 3 for the last 600 years (first block of Table 1). Events with $X_{\text {SPE56 }}<10$ cannot be reliably identified. We can also securely say that events with $X_{\text {SPE56 }}>20$ are not observed during that period (cf. Figure 4). In particular, a strong SPE related to the Carrington flare in 1859 contradicts these data.

We evaluate the probability $p$ of occurrence of such events, applying the Poisson distribution (assuming that SPEs are mutually independent). For example, for four events observed during 600 years, the probability is $p=0.0077_{-0.0045}^{+0.0073} \mathrm{yr}^{-1}$ ( $90 \%$ confidence interval), which is higher than the naively taken $4 / 600=0.0067 \mathrm{yr}^{-1}$. If no event with $F_{30}>2 \times$ $10^{10} \mathrm{~cm}^{-2}$ is observed over 600 years, the corresponding onesided $90 \%$ confidence interval is $p=0-0.0027 \mathrm{yr}^{-1}$, with median probability $0.0012 \mathrm{yr}^{-1}$ (once per 850 years).

The last 4-6 centuries cover the full range of solar activity, from Grand minima to the maximum (Usoskin 2008) serving as an archetype of the solar variability. Therefore, we expect that this result is consistent over longer scales. On the multimillennial scale (the Holocene-11 millennia), 19 candidates are identified (second block in Table 1) with $X_{\text {SPE56 }}=10-30$. A few candidates with $X_{\text {SPE56 }}>50$ were rejected by the crosscheck. This gives the average occurrence rate of such SPEs as roughly two per millennium. Moreover, we can securely say that during the Holocene there were no events with $X_{\text {SPE56 }}>$ 50-100, placing a strong upper limit on the SPE strength. We find no apparent relation between the occurrence of SPE candidates and the solar activity level (Usoskin et al. 2007).

We summarize our findings in a plot (Figure 5) of the integral probability of strong SPE occurrence. The $X$ - and $Y$-axes give 
the $F_{30}$ fluence and the probability of occurrence of SPEs with the $>30 \mathrm{MeV}$ proton annual fluence exceeding $F_{30}$ for different data sets. The measured annual fluences for the space era (1956-2008) are shown by open triangles (Shea \& Smart 1990; M. Shea 2012, private communication). Since the space era coincides with the unusually active Sun (Solanki et al. 2004), these probabilities may be higher than those for the typical medium activity Sun. The black triangle reflects the fact that no SPE with $F_{30}>10^{10} \mathrm{~cm}^{-2}$ was observed during 53 years. The open star corresponds to the four SPE candidates from the annual ${ }^{10}$ Be data (first block in Table 1), while the filled star corresponds to no SPE with $F_{30}>2 \times 10^{10} \mathrm{~cm}^{-2}$ found during 600 years. Open circles represent the candidates found in the rougher time series (second block in Table 1), grouped into four points: $F_{30}>1,1.5,2$, and $2.5 \times 10^{10} \mathrm{~cm}^{-2}$, respectively. Since the lower fluence value is probably underestimated because of the detection threshold, we ignore the first open circle, using instead the star-symbolled point from the annual ${ }^{10} \mathrm{Be}$ data sets. The filled circle corresponds to no event with $F_{30}>5 \times 10^{10} \mathrm{~cm}^{-2}$ found over 11 millennia. One can see that there is a break in the distribution at about $F_{30}=5 \times 10^{9} \mathrm{~cm}^{-2}$. This break (cf. McCracken et al. 2001; Hudson 2010) may be related to a streaming limit (Reames 1999) and/or limitations in the energetics of solar flares/coronal mass ejections (Fletcher et al. 2011). Our new results put a stronger constraint on the distribution. For example, an SPE with $X_{\text {SPE56 }}>20$ is expected only once per millennium, and no $X_{\text {SPE56 }}>50$ event is expected over tens of millennia. We try to fit the tail of the distribution $\left(F_{30}>7 \times 10^{9} \mathrm{~cm}^{-2}\right)$ by two simple models: exponential $\left(p \propto \exp \left(-0.33 \times 10^{-9} \cdot F_{30}\right)\right)$ and power law $\left(p \propto F_{30}^{-4}\right)$ - see the gray lines in the figure. The data do not allow us to distinguish between the two shapes.

We also compare our results with the average SEP flux over timescales of thousands to millions of years, estimated from lunar rocks, which range from $21-56\left(\mathrm{~cm}^{2} \mathrm{~s}\right)^{-1}$ (e.g., Reedy 1999; Nishiizumi et al. 2009). Time averaging of the results from Figure 5 using the above fits for the tail yields an average flux $\approx 38\left(\mathrm{~cm}^{2} \mathrm{~s}\right)^{-1}$, which is composed of about $35\left(\mathrm{~cm}^{2} \mathrm{~s}\right)^{-1}$ for the space era record (e.g., Shea \& Smart 2002; Reedy 2012) with an addition of $3.2\left(\mathrm{~cm}^{2} \mathrm{~s}\right)^{-1}$ due to the distribution tail based on cosmogenic isotope data (both exponent and power law give similar results), suggesting that extreme SPEs contribute only about $10 \%$ to the total SEP fluence. This is totally consistent with assessments based on lunar rocks, giving independent support to the present results. For an SPE with a softer energy spectrum, like SPE72, the tail addition would be 40 times greater (Section 2.2), leading to an average SEP flux $\approx 150\left(\mathrm{~cm}^{2} \mathrm{~s}\right)^{-1}$, in contradiction with the lunar rock data. Therefore, extreme SPEs found in the cosmogenic isotope records must have (on average) hard energy spectra.

\section{CONCLUSIONS}

We have evaluated the probability of occurrence of extreme SPEs based on data on the cosmogenic isotopes ${ }^{14} \mathrm{C}$ and ${ }^{10} \mathrm{Be}$ in terrestrial archives, spanning the timescale from centuries to 11 millennia (Figure 5). We identified four potential candidates for SPEs with $F_{30}=(1-1.5) \times 10^{10} \mathrm{~cm}^{-2}$ and show that no event with $F_{30}>2 \times 10^{10} \mathrm{~cm}^{-2}$ existed over the last 600 years using annually resolved ${ }^{10} \mathrm{Be}$ data. In particular, the extreme Carrington SPE of 1859 AD contradicts these data. From more roughly resolved data we identified 19 SPE candidates (Table 1) with $F_{30}=(1-3) \times 10^{10} \mathrm{~cm}^{-2}$, and clearly no event with
$F_{30}>5 \times 10^{10} \mathrm{~cm}^{-2}$, over the last 11,400 years. Two events, ca. $780 \mathrm{AD}$ and $1460 \mathrm{AD}$, appear in different series making them strong candidates for extreme SPEs. This gives a new strict observational constraint on the occurrence probability of extreme SPEs. Practical limits can be set as $F_{30} \approx 1,2-3$, and $5 \times 10^{10} \mathrm{~cm}^{-2}(10,20-30$, and 50 times greater than SPE56) for the occurrence probabilities of $10^{-2}, 10^{-3}$, and $10^{-4} \mathrm{yr}^{-1}$, respectively. The mean SEP flux is found as $\approx 40\left(\mathrm{~cm}^{2} \mathrm{~s}\right)^{-1}$, in agreement with estimates from lunar rocks. On average, extreme SPEs contribute about $10 \%$ to the total SEP fluence.

We note that the present result tends to represent an upper limit for SPE occurrence, since we explicitly assume that every peak in one data series, consistent with other series, is a signature of an SPE. This may be not exactly correct, as some of the peaks may be spurious. Accordingly, our results should be interpreted as a conservative upper limit on the SPE occurrence near Earth. Application of the results to the Sun is not straightforward, especially for more energetic events with low statistics, because the propagation of SEPs in interplanetary space may greatly affect the geo-efficiency of SPEs and, accordingly, their ability to become detectable in the cosmogenic isotope data series. Given these uncertainties, the present results should be considered with a precision of up to an order of magnitude.

We thank M. A. Shea for the data on observed annual SEP fluences. G.A.K. was partly supported by Program No. 22 of the Presidium RAS and also acknowledges support from the Academy of Finland and Suomalainen Tiedeakatemia (Väisälä foundation).

\section{REFERENCES}

Alanko-Huotari, K., Usoskin, I. G., Mursula, K., \& Kovaltsov, G. A. 2007, Adv. Space Res., 40, 1064

Bard, E., Raisbeck, G., Yiou, F., \& Jouzel, J. 1997, Earth Planet. Sci. Lett., 150, 453

Berggren, A.-M. 2009, PhD thesis, University of Uppsala, Sweden

Berggren, A.-M., Beer, J., Possnert, G., et al. 2009, Geophys. Res. Lett., 36, L11801

Damon, P. E., \& Peristykh, A. N. 2004, in AGU Geophysical Monograph Ser. 141, Solar Variability and its Effects on Climate, Geophysical Monograph 141, ed. J. M. Pap et al. (Washington DC: AGU), 237

Delaygue, G., \& Bard, E. 2011, Clim. Dyn., 36, 2201

Dreschhoff, G., \& Zeller, E. 1998, Sol. Phys., 177, 365

Field, C., Schmidt, G., Koch, D., \& Salyk, C. 2006, J. Geophys. Res., 111, D15107

Fletcher, L., Dennis, B. R., Hudson, H. S., et al. 2011, Space Sci. Rev., 159, 19

Heikkilä, U., Beer, J., \& Feichter, J. 2009, Atmos. Chem. Phys., 9, 515

Horiuchi, K., Uchida, T., Sakamoto, Y., et al. 2008, Quat. Geochronology, 3, 253

Hudson, H. S. 2010, Nature Phys., 6, 637

Kovaltsov, G. A., Mishev, A., \& Usoskin, I. G. 2012, Earth Planet. Sci. Lett., 337,114

Kovaltsov, G. A., \& Usoskin, I. G. 2010, Earth Planet. Sci. Lett., 291, 182

Lingenfelter, R. E., \& Hudson, H. S. 1980, in The Ancient Sun: Fossil Record in the Earth, Moon and Meteorites, ed. R. O. Pepin, J. A. Eddy, \& R. B. Merrill (Oxford: Pergamon), 69

McCracken, K., Dreschhoff, G., Zeller, E., Smart, D., \& Shea, M. 2001, J. Geophys. Res., 106, 21585

McCracken, K., McDonald, F., Beer, J., Raisbeck, G., \& Yiou, F. 2004, J. Geophys. Res., 109, 12103

Mewaldt, R. 2006, Space Sci. Rev., 124, 303

Meyer, P., Parker, E. N., \& Simpson, J. A. 1956, Phys. Rev., 104, 768

Miyake, F., Nagaya, K., Masuda, K., \& Nakamura, T. 2012, Nature, 486, 240

Nishiizumi, K., Arnold, J. R., Kohl, C. P., et al. 2009, Geochim. Cosmochim. Acta, 73, 2163

Raisbeck, G., Yiou, F., Jouzel, J., \& Petit, J. 1990, Phil. Trans. R. Soc. A, 330, 463 
Reames, D. V. 1999, Space Sci. Rev., 90, 413

Reedy, R. C. 1999, in Lunar and Planetary Institute Science Conference Abstracts, Vol. 30, Lunar and Planetary Inst. Technical Report, Contrib. LPI-000964, ed. LPI Editorial Board (Houston, TX: Lunar Planet. Inst.), art. 1643

Reedy, R. C. 2012, in Lunar and Planetary Institute Science Conference Abstracts, Vol. 43, Lunar and Planetary Inst. Technical Report, Contrib. LPI-000964, ed. LPI Editorial Board (Houston, TX: Lunar Planet. Inst.) art. 1285

Reimer, P. J., Baillie, M. G. L., Bard, E., et al. 2009, Radiocarbon, 51, 1111

Shea, M., \& Smart, D. 1990, Sol. Phys., 127, 297

Shea, M., \& Smart, D. 2002, Adv. Space Res., 29, 325

Shea, M., Smart, D., McCracken, K., Dreschhoff, G., \& Spence, H. 2006, Adv. Space Res., 38, 232

Smart, D. F., Shea, M. A., Spence, H. E., \& Kepko, L. 2006, Adv. Space Res., 37,1734

Solanki, S., Usoskin, I., Kromer, B., Schüssler, M., \& Beer, J. 2004, Nature, 431, 1084

Stuiver, M., \& Braziunas, T. F. 1993, Holocene, 3, 289
Tylka, A., \& Dietrich, W. 2009, in 31th International Cosmic Ray Conference, ed. M. Giller \& J. Szabelski (Lodź, Poland: Universal Academy Press), ICRC0273

Usoskin, I. G. 2008, Living Rev. Sol. Phys., 5, 3

Usoskin, I. G., Kovaltsov, G. A., Mironova, I. A., Tylka, A. J., \& Dietrich, W. F. 2011, Atmos. Chem. Phys., 11, 1979

Usoskin, I. G., Solanki, S. K., \& Kovaltsov, G. A. 2007, A\&A, 471, 301

Usoskin, I., Solanki, S., Kovaltsov, G., Beer, J., \& Kromer, B. 2006, Geophys. Res. Lett., 33, L08107

Vainio, R., Desorgher, L., Heynderickx, D., et al. 2009, Space Sci. Rev., 147, 187

Vonmoos, M., Beer, J., \& Muscheler, R. 2006, J. Geophys. Res., 111, A10105

Webber, W., Higbie, P., \& McCracken, K. 2007, J. Geophys. Res., 112, A10106

Wolff, E. W., Bigler, M., Curran, M. A. J., et al. 2012, Geophys. Res. Lett., 39, L08503

Wolff, E. W., Jones, A. E., Bauguitte, S. J.-B., \& Salmon, R. A. 2008, Atmos. Chem. Phys., 8, 5627

Yiou, F., Raisbeck, G. M., Baumgartner, S., et al. 1997, J. Geophys. Res., 102, 26783 